\title{
Correlation of clinical features and histopathological examination of ocular surface squamous neoplasia
}

\author{
P Ram Das', M M M Baig', Sara Sultana ${ }^{3}$, Mohammed Ather ${ }^{4, *}$ \\ 1,2 Assistant Professor, ${ }^{3}$ Senior Resident, ${ }^{4}$ Professor, Dept. of Ophthalmology, ${ }^{\mathbf{1}-3}$ Sarojini Devi Eye Hospital / Osmania Medical College, \\ Hyderabad Telangana, ${ }^{4}$ Sarojini Devi Eye Hospital / Gandhi Medical College, Secunderabad Telangana, India
}

*Corresponding Author:

Email: ather11258@gmail.com

\begin{abstract}
Aim: To study correlation between clinical features and histopathological exam of ocular surface squamous neoplasia.

Materials and Methods: This is a Prospective interventional study conducted at the department of Oculoplasty of Sarojini Devi eye hospital between January 2012 to June 2013. 54 patients of freshly diagnosed OSSN were included in the study. Cases which were treated or had recurrence were excluded from the study. All the cases were examined by an experienced Ophthalmologist using Slit lamp, Snellen's chart, Gonioscope and Direct Ophthalmoscope. Informed consent was obtained from all patients. After doing routine investigations like CBP, RBS, CT, BT, HIV, HbsAG patients were posted for excision biopsy of OSSN including 4mm of surrounding normal conjunctiva under local anaesthesia. Specimen was sent for Histopathological examination for diagnosis and margin clearance.

Results: Out of 54 cases 36 were males and 18 were females. 21 had dysplasia, 15 had carcinoma in situ, 18 had Squamous cell carcinoma. 33 cases had out door occupation and 21 had indoors occupation. 27 had nodular type, 15 had Leucoplakic type and 4 had diffuse type.

21 cases had corneal involvement. HPE grades 3 had mild dysplasia, 6 moderate dysplasia, 12 had severe dysplasia, 15 had carcinoma in situ, 18 had Squamous cell carcinoma. 15 cases were HIV positive and 39 cases were HIV negative.

Conclusion: Males out numbered females. Outdoor workers are more prone for OSSN. Nodular variety is commonest clinical type followed by Leucoplakic type and least common is diffuse type.
\end{abstract}

Keywords: OSSN, Dysplasia, Carcinoma in situ, Squamous cell carcinoma.

\section{Introduction}

The term Ocular surface squamous neoplasia (OSSN) was first described by Lee and Hirst in 1995 as an umbrella term that denotes spectrum of neoplasia originating from dysplasia to invasive squamous cell carcinoma. ${ }^{1}$

OSSN is growing pattern through out the world with higher frequency in equatorial region. Increase in incidence of OSSN is due to increase in Human immunodeficient virus and Human papilloma virus infections and exposure to Ultra violet rays. ${ }^{2}$

\section{Materials and Methods}

This is a Prospective interventional study carried out at the department of oculoplasty of Sarojini Devi eye hospital during January 2012 to June 2013. 54 freshly diagnosed cases of OSSN were included in the study. Cases which had treatment or came with recurrence were excluded from the study. Informed consent was obtained from all cases. All cases were examined by an experienced Ophthalmologist using Snellen's chart, Slit lamp, Gonioscope and Direct and indirect Ophthalmoscope. After doing routine investigations like CBP, RBS, CT, BT, HIV, HbsAG patients were posted for excision biopsy of OSSN including $4 \mathrm{~mm}$ of surrounding normal conjunctiva under local anaesthesia. Specimen was sent for Histopathological examination for diagnosis and margin clearance.

\section{Results}

Out of 54 cases 36 were males and 18 were females. 21 had dysplasia, 15 had carcinoma in situ, 18 had Squamous cell carcinoma. 33 cases had out door occupation and 21 had indoors occupation. According to Clinical classification of OSSN 27 had nodular type, 15 had Leucoplakic type and 4 had diffuse type.

21 cases had corneal involvement. According to HPE grades 3 had mild dysplasia, 6 moderate dysplasia, 12 had severe dysplasia, 15 had carcinoma in situ, 18 had Squamous cell carcinoma. 15 cases were HIV positive and 39 cases were HIV negative.

Age of the cases varied between 25-75 years with mean age of 39 years. $75 \%$ of the cases were in the age group of 41-60 years and they had higher grade lesions histopathologically like carcinoma in situ and Squamous cell carcinoma. This can be explained by generalised increase in incidence of malignancies in elderly population.

In 20-30 years age group had higher incidence of invasive carcinoma and they were HIV positive.

Clinically nodular type and Leucoplakic types had more number of carcinoma in situ and Squamous cell carcinoma and less number of dysplasias. Whereas diffuse variety had more number of dysplasias and less number of carcinoma in situ and squamous cell carcinoma.

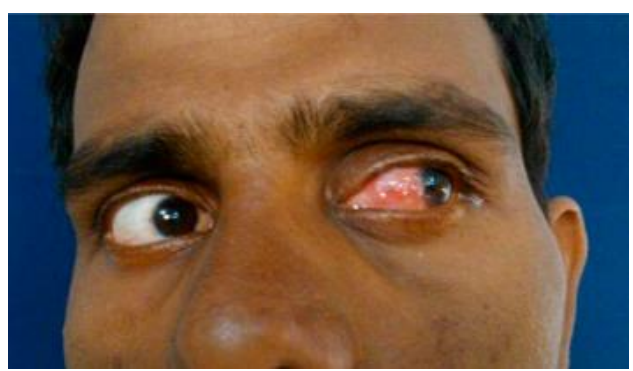

Fig. 1: Diffuse OSSN 


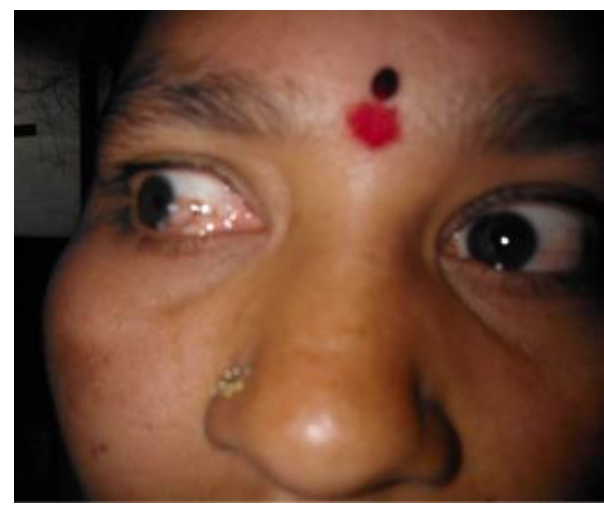

Fig. 2: Leukoplakic type

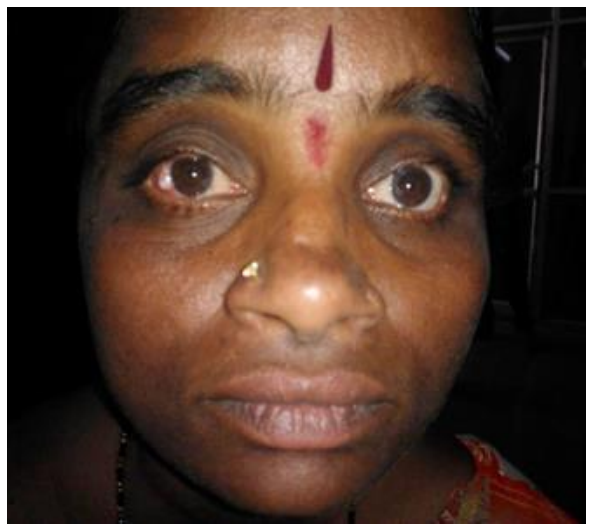

Fig. 3: Nodular types

\section{Discussion}

$\mathrm{T}$ Tiong et $\mathrm{al}^{3}$ studied 58 cases within the range of 2262 years with mean age of 35.8 years. Age group is consistent with present study.

Bascom Palmer eye institute ${ }^{4}$ studied 612 cases. $70 \%$ were males and $30 \%$ were females.

Rohit Bang et al had $63 \%$ males and $37 \%$ females. Male preponderance is also shown in this study.

Clinical type of OSSN: Study done by Jain Pradeep, Laxmi Chand et al, Rohit Bang et al and Bascom Palmer eye institute studies showed that nodular variety is the leading cause followed by Leucoplakic and diffuse type. All studies showed that nodular variety and Leucoplakic variety had higher incidence of carcinoma in situ and squamous cell carcinoma. This is in consistent with present study.

HIV Positivity: T Tiong et al HIV positive patients had invasive carcinoma 9 times greater than HIV negative patients. Spitzer MS et $\mathrm{al}^{5}$ in 2008 studied 53 cases of OSSN with HIV positive found 23 cases had invasive carcinoma, 21 had carcinoma in situ. This is consistent with present study wherein patients who were between 20-30 years age group had higher incidence of invasive carcinoma because of HIV positivity.

\section{Conclusion}

Males out numbered females. Outdoor workers are more prone for OSSN. Nodular variety is commonest clinical type followed by Leucoplakic type and least common is diffuse type. HIV positive patients with OSSN tends to have invasive carcinoma. Histopathologically more patients that is 33 had carcinoma in situ and Squamous cell carcinoma and 21 had only dysplastic changes.

\section{Conflict of Interest: Nil.}

\section{References}

1. Lee G A, Hirst LW. Retrospectivestudy of OSSN. Aust NZL J Ophth. 1997,25:269.

2. Winward KE. Conjunctival squamous cell carcinoma in Patients with HIV. AMJ of Ophth. 1989;107:554.

3. T Tiong. BJO. 2013;97:961-965.

4. Andrew Kao. Clinico pathological Correlation of OSSN. Ophthalmol. 2012;119(9)1773-1776.

5. Spitzer MS. OSSN as first manifestation of HIV. Clin Exp Ophthalmol. 2008;36:422-425.

How to cite this article: Das P R, Baig M. M. M, Sultana $S$, Ather M. Correlation of clinical features and histopathological examination of ocular surface squamous neoplasia. Int J Ocul Oncol Oculoplasty. 2018;4(4):172173. 